\title{
Factored Language Models and Generalized Parallel Backoff
}

\author{
Jeff A. Bilmes \\ Katrin Kirchhoff \\ SSLI-LAB, University of Washington, Dept. of Electrical Engineering \\ \{bilmes, katrin\}essli.ee.washington.edu
}

\begin{abstract}
We introduce factored language models (FLMs) and generalized parallel backoff (GPB). An FLM represents words as bundles of features (e.g., morphological classes, stems, data-driven clusters, etc.), and induces a probability model covering sequences of bundles rather than just words. GPB extends standard backoff to general conditional probability tables where variables might be heterogeneous types, where no obvious natural (temporal) backoff order exists, and where multiple dynamic backoff strategies are allowed. These methodologies were implemented during the JHU 2002 workshop as extensions to the SRI language modeling toolkit. This paper provides initial perplexity results on both CallHome Arabic and on Penn Treebank Wall Street Journal articles. Significantly, FLMs with GPB can produce bigrams with significantly lower perplexity, sometimes lower than highly-optimized baseline trigrams. In a multi-pass speech recognition context, where bigrams are used to create first-pass bigram lattices or N-best lists, these results are highly relevant.
\end{abstract}

\section{Introduction}

The art of statistical language modeling (LM) is to create probability models over words and sentences that tradeoff statistical prediction with parameter variance. The field is both diverse and intricate (Rosenfeld, 2000, Chen and Goodman, 1998; Jelinek, 1997; Ney et al., 1994), with many different forms of LMs including maximumentropy, whole-sentence, adaptive and cache-based, to name a small few. Many models are simply smoothed conditional probability distributions for a word given its preceding history, typically the two preceding words.

In this work, we introduce two new methods for language modeling: factored language model (FLM) and generalized parallel backoff (GPB). An FLM considers a word as a bundle of features, and GPB is a technique that generalized backoff to arbitrary conditional probability tables. While these techniques can be considered in isolation, the two methods seem particularly suited to each other - in particular, the method of GPB can greatly facilitate the production of FLMs with better performance.

\section{Factored Language Models}

In a factored language model, a word is viewed as a vector of $k$ factors, so that $w_{t} \equiv\left\{f_{t}^{1}, f_{t}^{2}, \ldots, f_{t}^{K}\right\}$. Factors can be anything, including morphological classes, stems, roots, and other such features in highly inflected languages (e.g., Arabic, German, Finnish, etc.), or data-driven word classes or semantic features useful for sparsely inflected languages (e.g., English). Clearly, a two-factor FLM generalizes standard class-based language models, where one factor is the word class and the other is words themselves. An FLM is a model over factors, i.e., $p\left(f_{t}^{1: K} \mid f_{t-1: t-n}^{1: K}\right)$, that can be factored as a product of probabilities of the form $p\left(f \mid f_{1}, f_{2}, \ldots, f_{N}\right)$. Our task is twofold: 1) find an appropriate set of factors, and 2) induce an appropriate statistical model over those factors (i.e., the structure learning problem in graphical models (Bilmes, 2003; Friedman and Koller, 2001)).

\section{Generalized Parallel Backoff}

An individual FLM probability model can be seen as a directed graphical model over a set of $N+1$ random variables, with child variable $F$ and $N$ parent variables $F_{1}$ through $F_{N}$ (if factors are words, then $F=W_{t}$ and $F_{i}=$ $\left.W_{t-i}\right)$. Two features make an FLM distinct from a standard language model: 1) the variables $\left\{F, F_{1}, \ldots, F_{N}\right\}$ can be heterogeneous (e.g., words, word clusters, morphological classes, etc.); and 2) there is no obvious natural (e.g., temporal) backoff order as in standard wordbased language models. With word-only models, backoff proceeds by dropping first the oldest word, then the next oldest, and so on until only the unigram remains. In $p\left(f \mid f_{1}, f_{2}, \ldots, f_{N}\right)$, however, many of the parent variables might be the same age. Even if the variables have differing seniorities, it is not necessarily best to drop the oldest variable first. 


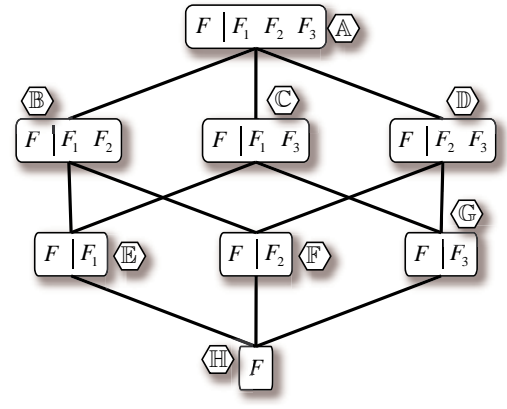

7 K L V H T X D WL R Q L V Q R Q 4 V WD Q G D U G R Q C where one may no longer sum over the factors $f$ only with counts greater than $\tau$. This is because $g \mathrm{~L} \mathrm{~V} \mathrm{QR} \mathrm{W} \mathrm{Q} \mathrm{H} \mathrm{F}$

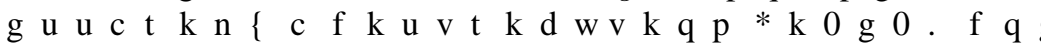
I R UH口 E DF NR I I Z H L J KW F R P S X WD WL R Q pensive for certain $g$ functions, but this appears not to be prohibitive as demonstrated in the next few sections.

Table 1: CallHome Arabic Results.

\begin{tabular}{|c|c|c|c|}
\hline LM & parents & backoff function/path(s) & $\mathrm{ppl}$ \\
\hline प J UDP & $w_{1}, w_{2}$ & ] WH P $[\mathbf{S}, \mathbb{R}]$ U D O & 173 \\
\hline ) $/ 0 \square \square J$ UDP & $w_{1}, w_{2}, m_{1}, s_{1}$ & ㅁ $[2,1,4,3]$ & 178 \\
\hline$* 3 \% \square) / 100$ & ${ }^{\prime} w \Psi, \mathrm{D} \mathrm{P}_{2}, m_{1}, s_{1}$ & $g_{1} /[2,1,(3,4), 3,4]$ & 166 \\
\hline ¿ & Ш L L & 7 WH P[B] R U D O & 175 \\
\hline S R R V NuI & $\begin{array}{l}w_{1}, m_{1} \\
\mathrm{E} w_{0} \mathbf{H}_{1}, s \mathbf{V} \mathrm{L}\end{array}$ & & $\begin{array}{l}173 \\
179\end{array}$ \\
\hline * $3 \% \square) / 00$ & $\downarrow w \Psi, \mathrm{DP}_{1}, s_{1}$ & $g_{1} /[1,(2,3), 2,3]$ & 167 \\
\hline
\end{tabular}

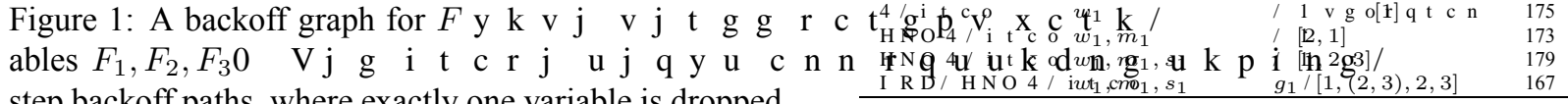
step backoff paths, where exactly one variable is dropped

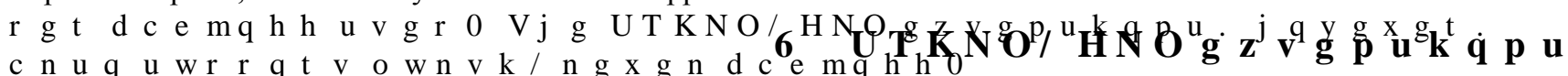
D O V R V X S S R U W P X O WL T O H Y H O E P F N Puring the recent 2002 JHU workshop (Kirchhoff et al.,
D

We introduce the notion of a backoff graph (Figure 1) to depict this issue, which shows the various backoff paths I UR P WK H DOOQ S DUHQWV F D unigram (bottom graph node). Many possible backoff paths could be taken. For example, when all variables are words, the path $\mathbb{A}-\mathbb{B}-\mathbb{E}-\mathbb{H} F$ R U U H V S R Q J U D P Z L WK V WD Q G D U G R O G H V W $\mathbb{A}-\mathbb{D}-\mathbb{G}-\mathbb{H} L \mathrm{~V}$ D U H Y H U V H $\mathrm{WL}$ P H E F D Q E H VHHQ DV D J H Q H D O L ] D WL modeling (Dupont and Rosenfeld, 1997) where factors consist of words and hierarchically derived word classes.

In our GPB procedure, either a single distinct path is chosen for each gram or multiple parallel paths are X V H G V L P X O WD Q H R X V O ए, Q off path(s) that are chosen are determined dynamically

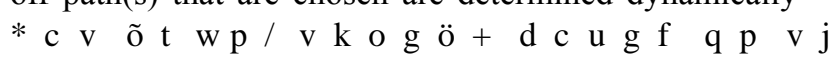
ables. For example, a path might consist of nodes $\mathbb{A}-(\mathbb{B C D})-(\mathbb{E} \mathbb{F})-\mathbb{G}$ where node $\mathbb{A}$ E D F N V R I I allel to the three nodes $\mathbb{B C D}$, node $\mathbb{B}$ backs off to nodes $(\mathbb{E} \mathbb{F}), \mathbb{C}$ backs off to $(\mathbb{E})$, and $\mathbb{D}$ backs off to $(\mathbb{F})$.

This can be seen as a generalization of the standard backoff equation. In the two parents case, this becomes:

$p_{G B O}\left(f \mid f_{1}, f_{2}\right)= \begin{cases}d_{N\left(f, f_{1}, f_{2}\right)} p_{M L}\left(f \mid f_{1}, f_{2}\right) \\ \alpha\left(f_{1}, f_{2}\right) g\left(f, f_{1}, f_{2}\right) & \text { if } N\left(f, f_{1}, f_{2}\right)>\tau\end{cases}$

where $d_{N\left(f, f_{1}, f_{2}\right)}$ is a standard discount (determining the smoothing method), $p_{M L} \mathrm{~L} \mathrm{~V}$ WK H P D [ L P hood distribution, $\alpha\left(f_{1}, f_{2}\right)$ are backoff weights, and

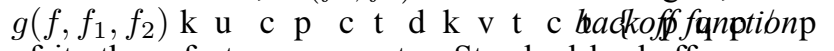
of its three factor arguments. Standard backoff occurs with $g\left(f, f_{1}, f_{2}\right)=p_{B O}\left(f \mid f_{1}\right)$, but the GPB procedures can be obtained by using different $g \square$ I X Q F WL R Q V ple, $g\left(f, f_{1}, f_{2}\right)=p_{B O}\left(f \mid f_{2}\right)$ corresponds to a different backoff path, and parallel backoff is obtained by using an appropriate $g$ (see below). As long as $g \mathrm{~L} \mathrm{~V} \mathrm{Q} \mathrm{R} \mathrm{Q} \square \mathrm{Q}$ WK H E DF NRI I Z H L K KV DUH GH

$$
\alpha\left(f_{1}, f_{2}\right)=\frac{1-\sum_{f: N\left(f, f_{1}, f_{2}\right)>\tau} d_{N\left(f, f_{1}, f_{2}\right)} p_{M L}\left(f \mid f_{1}, f_{2}\right)}{\sum_{f: N\left(f, f_{1}, f_{2}\right)<=\tau} g\left(f, f_{1}, f_{2}\right)}
$$

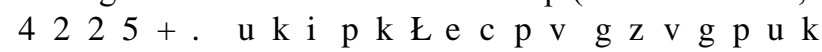
WUDU ) / 0 V DQG*3\%S URFHGXUHVD $7 \mathrm{KL}$

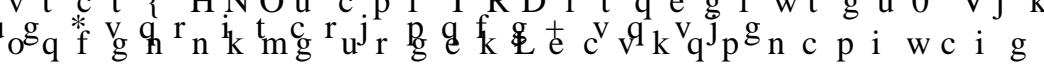
ferent backoff functions (19 in total) were implemented.

Other features include: 1) all SRILM smoothing methods

G V WR WW WU L L

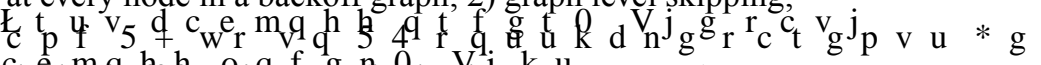

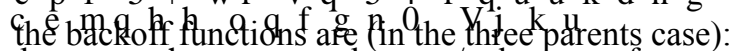

WL R Q R I O D WWL F H E DVHG ODQ J X D J H

$$
g\left(f, f_{1}, f_{2}, f_{3}\right)=p_{G B O}\left(f \mid f_{\ell_{1}}, f_{\ell_{2}}\right)
$$

where

$$
\left(\ell_{1}, \ell_{2}\right)=\quad \operatorname{argmax} \quad p_{G B O}\left(f \mid f_{m_{1}}, f_{m_{2}}\right)
$$

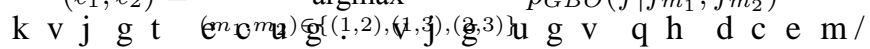

(call this $g_{1}$ ) or alternatively, where

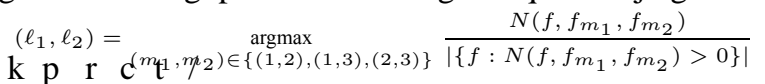

(call this $g_{2}$ ) where $N()$ L V WK H F R X Q W I X Q F WL R Q P H Q WH G E D F NR I I I X Q F WL R Q V L Q F OX G H malized) counts/backoff probabilities, products, sums, mins, maxs, (weighted) averages, and geometric means.

\section{ㄴ 5 H V X O WV}

* $3 \%$ ) / $0 \mathrm{~V} \mathrm{Z} \mathrm{HUH} \mathrm{DS} \mathrm{S} \mathrm{OL} \mathrm{HG} \mathrm{WR} \mathrm{WZ} \mathrm{R} \mathrm{F} \mathrm{R} \mathrm{U}$ X P $9[\mathrm{~L} I N$ N DQG WUL J U D P O D Q J X D J H P R GHOV , Q WH $\mathrm{HJ}_{\mathrm{L}} \mathrm{Q}_{\mathrm{G}} \mathrm{HLU}^{Y} \mathrm{H}_{\mathrm{V}} \mathrm{D}^{3}$ E L J U DP' D O DQJ X D J H P R G history that includes information from no longer than one

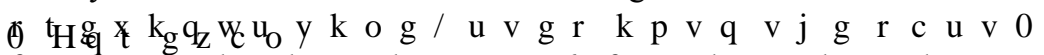
G H WH U P L Q L V WL F D O \ GHUL Y DE OH I UR P include both the previous words and previous factors as

HD RWW U U ） UR P D GHF R G L Q J V WDWH Quch bigran wouldbe relatively cheap.

, Q \& D O O R P H \$ U D L F I Z R UG V D U H D F terministically derived factors: morphological class (M), 
Table 2: Penn Treebank WSJ Results.

\begin{tabular}{lllc} 
LM & parents & Backoff function/path(s) & ppl ( \pm std. dev. $)$ \\
\hline 3-gram & $w_{1}, w_{2}$ & $-/$ temporal $[2,1]$ & $258( \pm 1.2)$ \\
2-gram & $w_{1}$ & $-/$ temporal $[1]$ & $320( \pm 1.3)$ \\
GPB-FLM 2-gram A & $w_{1}, d_{1}, t_{1}$ & $g_{2} /[(1,2,3),(1,2),(2,3),(3,1), 1,2,3]$ & $266( \pm 1.1)$ \\
GPB-FLM 2-gram B & $w_{1}, d_{1}, f_{1}$ & $g_{2} /[2,1]$ & $276( \pm 1.3)$ \\
GPB-FLM 2-gram C & $w_{1}, d_{1}, c_{1}$ & $g_{2} /[1,(2,3), 2,3]$ & $275( \pm 1.2)$ \\
\hline
\end{tabular}

stems $(\mathrm{S})$, roots $(\mathrm{R})$, and patterns $(\mathrm{P})$. Training data consisted of official training portions of the LDC CallHome ECA corpus plus the CallHome ECA supplement (100 conversations). For testing we used the official 1996 evaluation set. Results are given in Table 1 and show perplexity for: 1) the baseline 3-gram; 2) a FLM 3-gram using morphs and stems; 3 ) a GPB-FLM 3-gram using morphs, stems and backoff function $\left.g_{1} ; 4\right)$ the baseline 2-gram; 5) an FLM 2-gram using morphs; 6) an FLM 2-gram using morphs and stems; and 7) an GPB-FLM 2-gram using morphs and stems. Backoff path(s) are depicted by listing the parent number(s) in backoff order. As can be seen, the FLM alone might increase perplexity, but the GPB-FLM decreases it. Also, it is possible to obtain a 2-gram with lower perplexity than the optimized baseline 3-gram.

The Wall Street Journal (WSJ) data is from the Penn Treebank 2 tagged ('88-'89) WSJ collection. Word and POS tag information $\left(T_{t}\right)$ was extracted. The sentence order was randomized to produce 5-fold crossvalidation results using (4/5)/(1/5) training/testing sizes. Other factors included the use of a simple deterministic tagger obtained by mapping a word to its most frequent tag $\left(F_{t}\right)$, and word classes obtained using SRILM's ngram-class tool with $50\left(C_{t}\right)$ and $500\left(D_{t}\right)$ classes. Results are given in Table 2 The table shows the baseline 3-gram and 2-gram perplexities, and three GPB-FLMs. Model A uses the true by-hand tag information from the Treebank. To simulate conditions during first-pass decoding, Model B shows the results using the most frequent tag, and Model $\mathrm{C}$ uses only the two data-driven word classes. As can be seen, the bigram perplexities are significantly reduced relative to the baseline, almost matching that of the baseline trigram. Note that none of these reduced perplexity bigrams were possible without using one of the novel backoff functions.

\section{Discussion}

The improved perplexity bigram results mentioned above should ideally be part of a first-pass recognition step of a multi-pass speech recognition system. With a bigram, the decoder search space is not large, so any appreciable LM perplexity reductions should yield comparable word error reductions for a fixed set of acoustic scores in a firstpass. For N-best or lattice generation, the oracle error should similarly improve. The use of an FLM with GPB in such a first pass, however, requires a decoder that supports such language models. Therefore, FLMs with GPB will be incorporated into GMTK (Bilmes, 2002), a general purpose graphical model toolkit for speech recognition and language processing. The authors thank Dimitra Vergyri, Andreas Stolcke, and Pat Schone for useful discussions during the JHU'02 workshop.

\section{References}

[Bilmes2002] J. Bilmes. 2002. The GMTK documentation. http://ssli.ee.washington.edu/ ^bilmes/gmtk.

[Bilmes2003] J. A. Bilmes. 2003. Graphical models and automatic speech recognition. In R. Rosenfeld, M. Ostendorf, S. Khudanpur, and M. Johnson, editors, Mathematical Foundations of Speech and Language Processing. SpringerVerlag, New York.

[Chen and Goodman1998] S. F. Chen and J. Goodman. 1998. An empirical study of smoothing techniques for language modeling. Technical Report Tr-10-98, Center for Research in Computing Technology, Harvard University, Cambridge, Massachusetts, August.

[Dupont and Rosenfeld1997] P. Dupont and R. Rosenfeld. 1997. Lattice based language models. Technical Report CMU-CS-97-173, Carnegie Mellon University, Pittsburgh, PA 15213, September.

[Friedman and Koller2001] N. Friedman and D. Koller. 2001. Learning Bayesian networks from data. In NIPS 2001 Tutorial Notes. Neural Information Processing Systems, Vancouver, B.C. Canada.

[Jelinek1997] F. Jelinek. 1997. Statistical Methods for Speech Recognition. MIT Press.

[Kirchhoff et al.2003] K. Kirchhoff et al 2003. Novel approaches to arabic speech recognition: Report from the 2002 johns-hopkins summer workshop. In Proc. IEEE Intl. Conf. on Acoustics, Speech, and Signal Processing, Hong Kong.

[Ney et al.1994] H. Ney, U. Essen, and R. Kneser. 1994. On structuring probabilistic dependencies in stochastic language modelling. Computer Speech and Language, 8:1-38.

[Rosenfeld2000] R. Rosenfeld. 2000. Two decades of statistical language modeling: Where do we go from here? Proceedings of the IEEE, 88(8).

[Stolcke2002] A. Stolcke. 2002. SRILM- an extensible language modeling toolkit. In Proc. Int. Conf. on Spoken Language Processing, Denver, Colorado, September. 